\title{
Notes on interval halving procedure for periodic and two-point problems
}

\author{
András Rontó ${ }^{*}$, Miklós Rontó ${ }^{2}$ and Nataliya Shchobak ${ }^{3,4}$
}

"Correspondence: ronto@math.cas.cz

${ }^{1}$ Institute of Mathematics, Academy of Sciences of Czech Republic, 22 Žižkova St., Brno, 616 62, Czech Republic

Full list of author information is available at the end of the article

\begin{abstract}
We continue our study of constructive numerical-analytic schemes of investigation of boundary problems. We simplify and improve the recently suggested interval halving technique allowing one to essentially weaken the convergence conditions.

MSC: Primary 34B15

Keywords: periodic solution; two-point problem; Lyapunov-Schmidt reduction; determining equation; parametrisation; periodic successive approximations; numerical-analytic method; Cesari method; interval halving
\end{abstract}

\section{Introduction}

The present note is a continuation of [1] and deals with a constructive approach to the investigation of two-point boundary value problems. The approach is numerical-analytic $[2,3]$ in the sense that, although part of the computation is carried out analytically, the final stage of the method involves a numerical analysis of certain equations usually referred to as determining, or bifurcation, equations. This scheme of Lyapunov-Schmidt type $[4,5]$ reminds one of the shooting method on first glance, but there are several essential differences [1].

We consider the periodic boundary value problem

$$
\begin{aligned}
& u^{\prime}(t)=f(t, u(t)), \quad t \in[0, p], \\
& u(0)=u(p),
\end{aligned}
$$

where $p \in(0, \infty), f:[0, p] \times \mathbb{R}^{n} \rightarrow \mathbb{R}^{n}$ satisfies the Carathéodory conditions, and a solution is an absolutely continuous vector function satisfying (1) almost everywhere on $[0, p]$. Our main assumption till the end of the paper is that there exist a certain matrix $K$ and a bounded closed set $\Omega \subset \mathbb{R}^{n}$ such that $f(t, \cdot) \in \operatorname{Lip}_{K}(\Omega)$ for a.e. $t \in[0, p]$. Here and below, given a square matrix $K$ with non-negative entries, $\operatorname{Lip}_{K}(\Omega)$ stands for the set of functions $g: \Omega \rightarrow \mathbb{R}^{n}$ satisfying the componentwise Lipschitz condition

$$
\left|g\left(z_{1}\right)-g\left(z_{2}\right)\right| \leq K\left|z_{1}-z_{2}\right|
$$

for all $z_{1}$ and $z_{2}$ from $\Omega$. In (3) and all similar relations that will appear below, the symbols $\leq$ and $|\cdot|$ are understood componentwise.

(c) 2014 Rontó et al.; licensee Springer. This is an Open Access article distributed under the terms of the Creative Commons Attribution License (http://creativecommons.org/licenses/by/2.0), which permits unrestricted use, distribution, and reproduction in any medium, provided the original work is properly credited. 
In its original form (see, e.g., [2, 3] for references), the numerical-analytic approach that we are dealing with suggests one to look for a solution of (1), (2) among the limit functions of certain $n$-parametric family of sequences possessing property (2) (see, e.g., [2, 3]). Given an arbitrary vector $\xi$, consider the sequence of functions defined by the recurrence relation

$$
u_{m}(t, \xi):=\xi+\int_{0}^{t} f\left(s, u_{m-1}(s, \xi)\right) d s-\frac{t}{p} \int_{0}^{p} f\left(s, u_{m-1}(s, \xi)\right) d s, \quad t \in[0, p]
$$

with $m=1,2, \ldots$ and $u_{0}(t, \xi):=\xi, t \in[0, p]$. Clearly, each of functions (4) satisfies the periodic boundary condition (2). If one establishes the existence of the limit

$$
u_{\infty}(\cdot, \xi):=\lim _{m \rightarrow \infty} u_{m}(\cdot, \xi), \quad \xi \in \Omega
$$

with a certain $\Omega \subset D$, one finds out that the existence of a solution $u(\cdot)$ of the periodic problem (1), (2) with the value at zero lying in $\Omega$ is equivalent to the solvability of the equation

$$
\int_{0}^{p} f\left(s, u_{\infty}(s, \xi)\right) d s=0
$$

with respect to the unknown vector $\xi$. This leads to a Lyapunov-Schmidt type reduction of the periodic problem (see [1,3] for more details), which is known to be applicable on the assumption that

$$
f(t, \cdot) \in \operatorname{Lip}_{K}(D) \quad \text { for a.e. } t \in[0, p]
$$

with $p K$ small enough and $D$ satisfying the condition

$$
D\left(\frac{T}{4} \delta_{D}(f)\right) \neq \varnothing
$$

where

$$
\delta_{D}(f):=\max \left\{\delta_{[0, p / 2], D}(f), \delta_{[p / 2, p], D}(f)\right\}
$$

and $\delta_{J, V}(f):=\max _{(t, \xi) \in J \times V} f(t, \xi)-\min _{(t, \xi) \in J \times V} f(t, \xi)$ for any compact $V \subseteq D$ and $J \subseteq[0, p]$. In (7), $D(\varrho)$ is the $\varrho$-core of $D$ defined as

$$
D(\varrho):=\{z \in D: B(z, \varrho) \subset D\}
$$

for any non-negative vector $\varrho$, where

$$
B(z, \varrho):=\left\{\xi \in \mathbb{R}^{n}:|z-\xi| \leq \varrho\right\}
$$

is the (componentwise) $\varrho$-neighbourhood of $z$. Note that $\Omega$ involved in (5) is actually equal to $D(\varrho)$ in that case. Examples showing how $D(\varrho)$ can look like for different $D$ can be seen on Figure 1. 


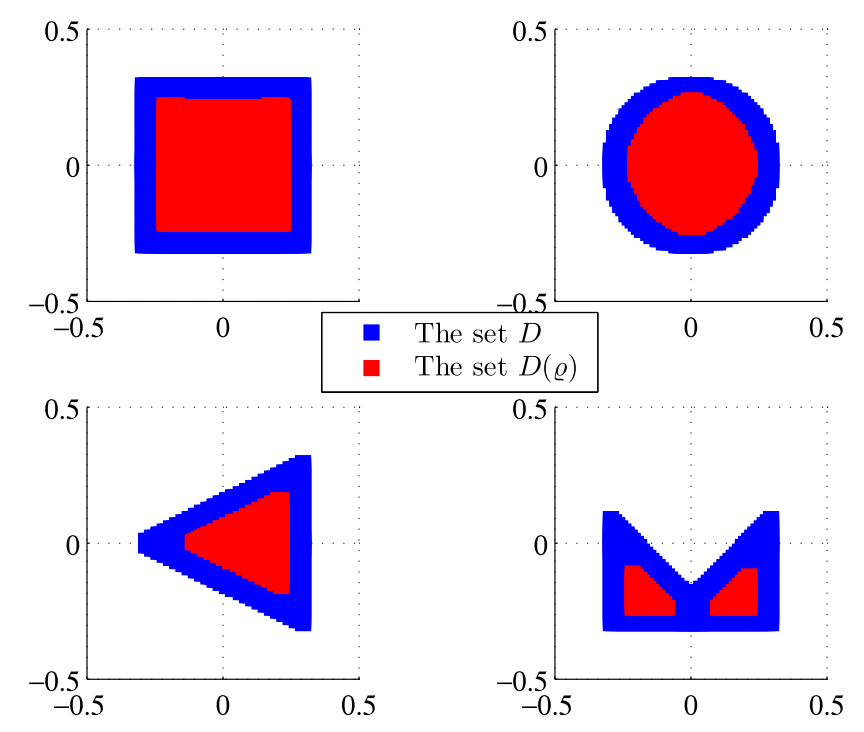

Figure 1 The set $D(\varrho)$ in examples, $\varrho=\operatorname{col}(1 / 12,1 / 18)$.

The main limitation of this approach is that, in order to guarantee the convergence, one has to assume a certain smallness of the eigenvalues of the matrix $p K$. It was shown, in particular, in [6] that the method based upon sequence (4) is applicable provided that

$$
r(K)<\frac{1}{\gamma_{0} p},
$$

where

$$
\gamma_{0}:=\frac{3}{10}
$$

Moreover, as is seen from condition (7), the set $D$ where (6) holds should be wide enough (in particular, such that $\operatorname{diam} D \geq \frac{p}{2} \delta_{D}(f)$, with the natural componentwise definition of a vector-valued diameter of a set).

As the recent paper [1] shows, the limitation can be overcome by noticing that the quantity which is assumed be small enough is always proportional to the length of the interval. A natural interval halving technique then allows one to produce a version of the scheme where (11) is replaced by the condition

$$
r(K)<\frac{2}{\gamma_{0} p}
$$

and, thus, weakened by half. A similar improvement is also achieved in relation to condition (7), which is replaced by the assumption that

$$
D\left(\frac{T}{8} \delta_{D}(f)\right) \neq \varnothing .
$$

Clearly, the transition to (13) weakens (7) by half.

Here, we modify the scheme of [1] so that its substantiation is simplified and, in particular, replace (7) by an assumption which is more transparent and, generally speaking, less 
restrictive. Indeed, the idea to start from a set $D$ where the nonlinearity is known to be Lipschitzian and look for its suitable subset $D(\varrho)$ that could potentially contain initial values of periodic solutions is somewhat unnatural because, in any case, it is the initial values that are of major interest, the regularity assumptions for the equation being only technical assumptions induced by the method. Instead of doing so, which used to be the case in [1] and in all the previous works, it is, however, more logical to choose a closed bounded set $\Omega \subset \mathbb{R}^{n}$, where one expects to find initial values of the solution, and to assume that the nonlinearity is Lipschitzian on a suitable $\tilde{\Omega} \supset \Omega$, with $\tilde{\Omega}$ only as large as the method requires. It is not difficult to see that the argument of [1] then leads us to the choice $\tilde{\Omega}:=\Omega_{\varrho}$, where $\Omega_{\varrho}$ is the $\varrho$-neighbourhood of $\Omega$ in the sense that

$$
\Omega_{\varrho}:=\bigcup_{\xi \in \Omega} B(\xi, \varrho)
$$

where the symbol $B(\xi, \varrho)$ stands for the $\varrho$-neighbourhood of a vector $\xi$ (recall that the relations in (10) are componentwise). Besides its more natural character, the use of the pair of sets $\left(\Omega_{\varrho}, \Omega\right)$ is also advantageous in contrast to $(D, D(\varrho))$ because, geometrically, $D(\varrho)$ does not necessarily copy the shape of $D$ (see Figures 2 and 3 for examples where $D$ and the corresponding $D(\varrho)$ with $\varrho=\left(\begin{array}{l}\varrho_{1} \\ \varrho_{2}\end{array}\right)$ gradually increasing are represented, respectively, by the blue and red regions). In fact, the operations of taking $\varrho$-core and $\varrho$-neighbourhood do not commute: the equality in the inclusion

$$
(D(\varrho))_{\varrho} \subset D
$$

is, in general, not true, whereas one obviously has

$$
\Omega_{\varrho}(\varrho)=\Omega
$$

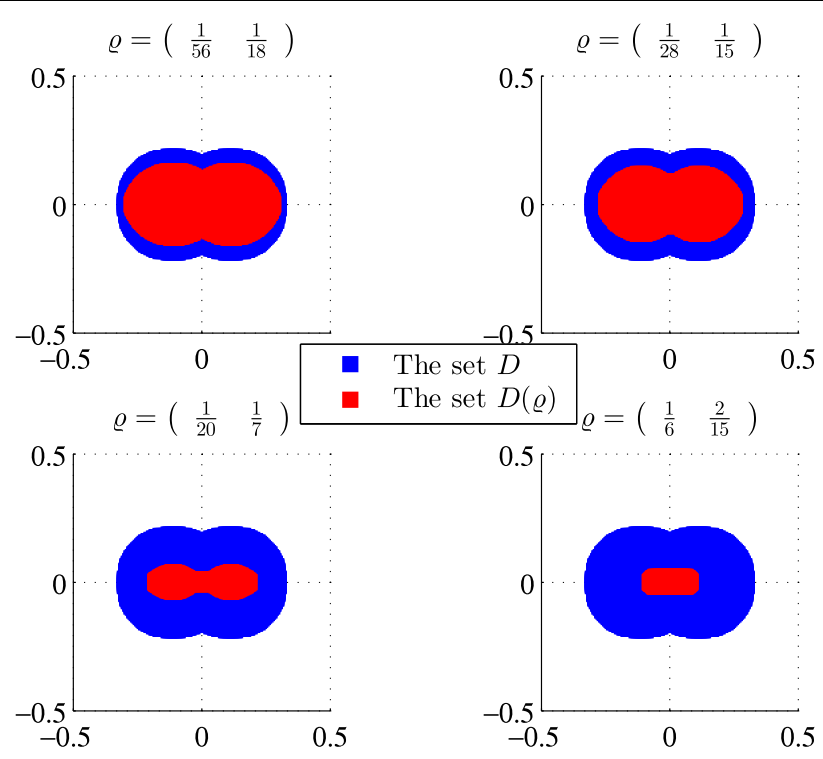

Figure 2 The shape of $D(\varrho)$ may differ significantly for various values of $\varrho$. 


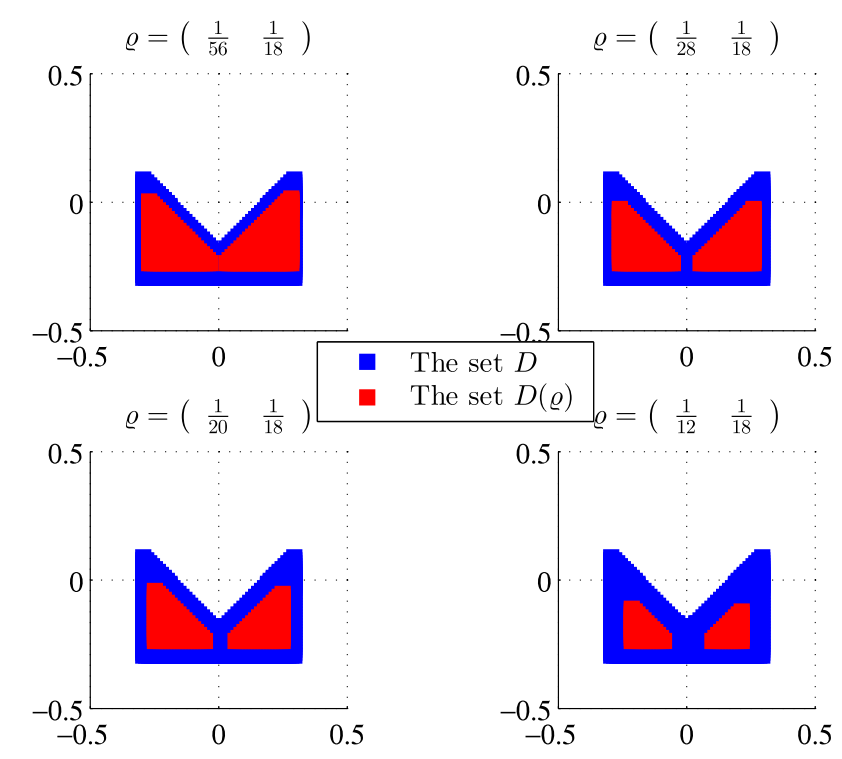

Figure 3 An example of $D$ with $D(\varrho)$ disconnected for certain $\varrho$.

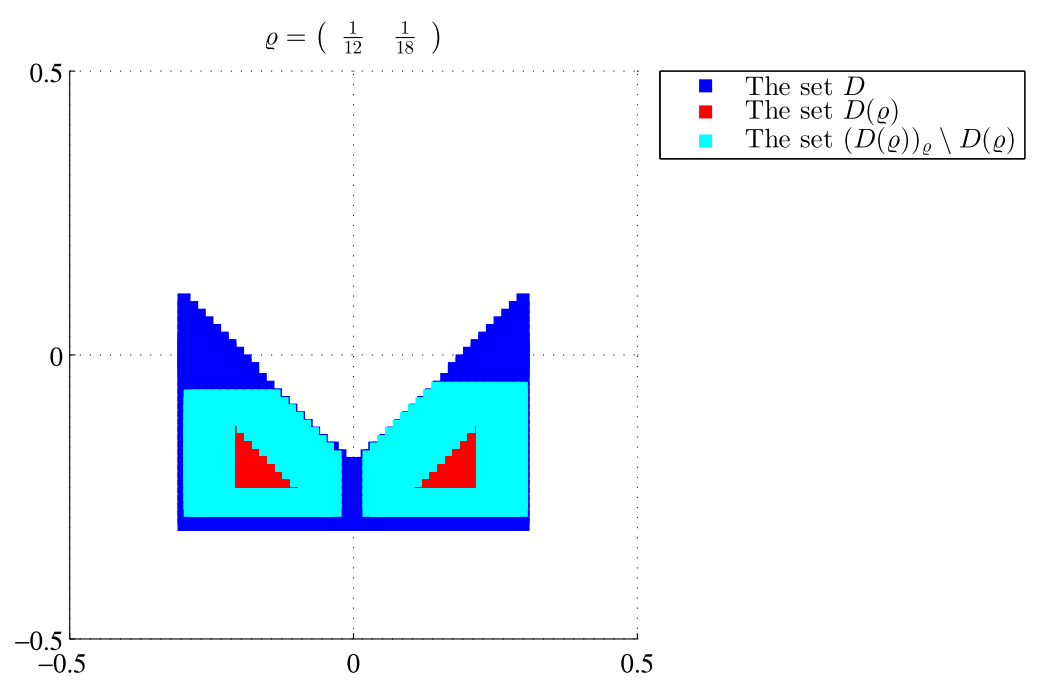

Figure 4 An example of the set $D$ illustrating the strict inclusion in (15).

for any $\varrho$. The strict inclusion in (15) holds, in particular, in the example from Figure 4, where the points of the sets $D(\varrho), D$ and $(D(\varrho))_{\varrho}$ for several values of $\varrho=\left(\begin{array}{l}\varrho_{1} \\ \varrho_{2}\end{array}\right), \varrho_{1} \leq \varrho_{2}$, are plotted in red, blue and cyan, respectively. In that example, by choosing $\Omega$ to be the red region, one should then widen it for the technical purposes related to the method up to the cyan one, and not the blue one. A comparison of (15) and (16) confirms the advantage of assuming conditions of type (6) on $\Omega_{\varrho}$. Several examples of domains $\Omega$ and the corresponding sets $\Omega_{\varrho}$ can be seen on Figure 5 .

Using the $\left(\Omega_{\varrho}, \Omega\right)$ setting, we further reformulate the scheme of the method further by removing certain unnecessary technicalities so that both the setting and the overall analysis are simplified. The new formulation, in particular, makes it particularly easy to adopt 


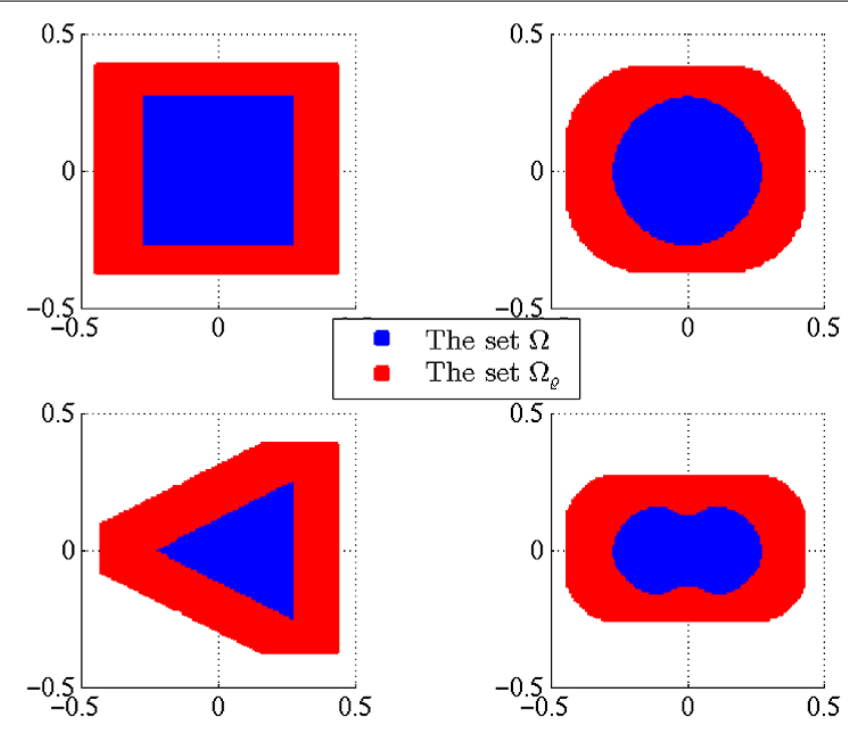

Figure 5 The set $\Omega_{\varrho}$ in examples, $\varrho=\operatorname{col}(1 / 8,1 / 16)$.

the approach to problems with two-point boundary conditions different from the periodic ones, which technique is also outlined in what follows.

\section{Construction of iterations and proof of convergence}

Thus, let us fix a closed bounded set $\Omega \subset \mathbb{R}^{n}$, where the initial values of solutions of problem (1), (2) will be looked for. Without loss of generality, we shall choose $\Omega$ to be convex. Let $\xi$ and $\eta$ be arbitrary vectors from $\Omega$. Let us put

$$
\begin{aligned}
& x_{0}(t, \xi, \eta):=\left(1-\frac{2 t}{p}\right) \xi+\frac{2 t}{p} \eta, \quad t \in[0, p / 2], \\
& y_{0}(t, \xi, \eta):=2\left(1-\frac{t}{p}\right) \eta+\left(\frac{2 t}{p}-1\right) \xi, \quad t \in[p / 2, p],
\end{aligned}
$$

and define the recurrence sequences of functions $x_{m}:[0, p / 2] \times \Omega^{2} \rightarrow \mathbb{R}^{n}$ and $y_{m}$ : $[p / 2, p] \times \Omega^{2} \rightarrow \mathbb{R}^{n}, m=0,1, \ldots$, according to the formulae

$$
\begin{aligned}
x_{m}(t, \xi, \eta):= & x_{0}(t, \xi, \eta)+\int_{0}^{t} f\left(s, x_{m-1}(s, \xi, \eta)\right) d s \\
& -\frac{2 t}{p} \int_{0}^{\frac{p}{2}} f\left(s, x_{m-1}(s, \xi, \eta)\right) d s, \quad t \in[0, p / 2], \\
y_{m}(t, \xi, \eta):= & y_{0}(t, \xi, \eta)+\int_{\frac{p}{2}}^{t} f\left(s, y_{m-1}(s, \xi, \eta)\right) d s \\
& -\left(\frac{2 t}{p}-1\right) \int_{\frac{p}{2}}^{p} f\left(s, y_{m-1}(s, \xi, \eta)\right) d s, \quad t \in[p / 2, p],
\end{aligned}
$$

where $m \geq 0$. One arrives at formulae (17), (18) directly when choosing $x_{0}(\cdot, \xi, \eta)$ and $y_{0}(\cdot, \xi, \eta)$ as linear functions on the appropriate intervals satisfying the equalities

$$
x_{0}(0, \xi, \eta)=\xi, \quad x_{0}\left(\frac{p}{2}, \xi, \eta\right)=\eta
$$




$$
y_{0}\left(\frac{p}{2}, \xi, \eta\right)=\eta, \quad y_{0}(p, \xi, \eta)=\xi
$$

The considerations in [1] concerning the auxiliary parametrised problems (4.1), (4.2) and (4.3), (4.4) can be omitted. Clearly, (17) and (18) are the simplest choice of functions satisfying (21) and (22).

The form of sequences (19), (20) is motivated by the following proposition.

Proposition 1 Let $(\xi, \eta) \in \Omega^{2}$ be fixed. If the limits $x_{\infty}(\cdot, \xi, \eta)$ and $y_{\infty}(\cdot, \xi, \eta)$ of sequences (19) and (20), respectively, exist uniformly on $\left[0, \frac{1}{2} p\right]$ and $\left[\frac{1}{2} p, p\right]$, then:

1. The function $x_{\infty}(\cdot, \xi, \eta)$ has the property

$$
x_{\infty}\left(\frac{p}{2}, \xi, \eta\right)-x_{\infty}(0, \xi, \eta)=\eta-\xi
$$

and is the unique solution of the initial value problem

$$
\begin{aligned}
& x^{\prime}(t)=f(t, x(t))+\frac{2}{p} \Xi(\xi, \eta), \quad t \in[0, p / 2], \\
& x(0)=\xi
\end{aligned}
$$

where

$$
\Xi(\xi, \eta):=\eta-\xi-\int_{0}^{\frac{p}{2}} f\left(\tau, x_{\infty}(\tau, \xi, \eta)\right) d \tau
$$

2. The function $y_{\infty}(\cdot, \xi, \eta)$ has the property

$$
y_{\infty}(p, \xi, \eta)-y_{\infty}\left(\frac{p}{2}, \xi, \eta\right)=\xi-\eta
$$

and is the unique solution of the initial value problem

$$
\begin{aligned}
& y^{\prime}(t)=f(t, y(t))+\frac{2}{p} \mathrm{H}(\xi, \eta), \quad t \in[p / 2, p], \\
& y\left(\frac{p}{2}\right)=\eta
\end{aligned}
$$

where

$$
\mathrm{H}(\xi, \eta):=\xi-\eta-\int_{\frac{p}{2}}^{p} f\left(\tau, y_{\infty}(\tau, \xi, \eta)\right) d \tau
$$

The proposition stated above, which is an easy consequence of the definitions of the functions $x_{m}:[0, p / 2] \times \Omega^{2} \rightarrow \mathbb{R}^{n}$ and $y_{m}:[p / 2, p] \times \Omega^{2} \rightarrow \mathbb{R}^{n}, m=0,1, \ldots$, suggests one to consider the function $u_{\infty}(\cdot, \xi, \eta):[0, p] \rightarrow \mathbb{R}^{n}$ introduced according to the formula

$$
u_{\infty}(t, \xi, \eta):= \begin{cases}x_{\infty}(t, \xi, \eta) & \text { if } t \in[0, p / 2] \\ y_{\infty}(t, \xi, \eta) & \text { if } t \in(p / 2, p]\end{cases}
$$


for all $\xi$ and $\eta$ from $\Omega$ and look for solutions of problem (1), (2) in the form $u_{\infty}(\cdot, \xi, \eta)$. Note that, as follows immediately from (23), (25) and (29),

$$
x_{\infty}\left(\frac{p}{2}, \xi, \eta\right)=y_{\infty}\left(\frac{p}{2}, \xi, \eta\right)
$$

and, therefore, the function $u_{\infty}(\cdot, \xi, \eta)$ is continuous on $[0, p]$ for any $(\xi, \eta) \in \Omega^{2}$. The use of this function, however, requires the knowledge of the fact that $x_{\infty}(\cdot, \xi, \eta)$ and $y_{\infty}(\cdot, \xi, \eta)$ are well defined for $(\xi, \eta) \in \Omega^{2}$.

Introduce the functions

$$
\bar{\alpha}_{1}(t):=\frac{2}{p} t(p-t), \quad t \in[0, p / 2]
$$

and

$$
\overline{\bar{\alpha}}_{1}(t):=\frac{1}{2 p}(p-2 t)(2 t-3 p), \quad t \in[p / 2, p] .
$$

Theorem 2 If there exists a non-negative vector $\varrho$ with the property

$$
\varrho \geq \frac{p}{8} \delta_{\Omega_{\varrho}}(f)
$$

such that $f(t, \cdot) \in \operatorname{Lip}_{K}\left(\Omega_{\varrho}\right)$ for a.e. $t \in[0, p]$ with a certain $K$ and

$$
r(K)<\frac{2}{\gamma_{0} p}
$$

then, for all fixed $(\xi, \eta) \in \Omega^{2}$, the sequence $\left\{x_{m}(\cdot, \xi, \eta): m \geq 0\right\}$ (resp., $\left.\left\{y_{m}(\cdot, \xi, \eta): m \geq 0\right\}\right)$ converges to a limit function $x_{\infty}(\cdot, \xi, \eta)$ (resp., $\left.y_{\infty}(\cdot, \xi, \eta)\right)$ uniformly in $t \in[0, p / 2]$ (resp., $t \in[p / 2, p])$, and the following estimates hold:

$$
\left|x_{m}(\cdot, \xi, \eta)-x_{\infty}(t, \xi, \eta)\right| \leq \frac{\bar{\alpha}_{1}(t)}{2^{m+1}}\left(\gamma_{0} p K\right)^{m}\left(\mathbf{1}_{n}-\frac{\gamma_{0} p}{2} K\right)^{-1} \delta_{[0, p / 2], \Omega_{\varrho}}(f)
$$

for all $t \in[0, p / 2]$ and

$$
\left|y_{m}(\cdot, \xi, \eta)-y_{\infty}(t, \xi, \eta)\right| \leq \frac{\overline{\bar{\alpha}}_{1}(t)}{2^{m+1}}\left(\gamma_{0} p K\right)^{m}\left(\mathbf{1}_{n}-\frac{\gamma_{0} p}{2} K\right)^{-1} \delta_{[p / 2, p], \Omega_{\varrho}}(f)
$$

for all $t \in[p / 2, p]$ and $m \geq 3$.

In estimates (36) and (37), the symbol $\mathbf{1}_{n}$ stands for the unit matrix of dimension $n$. Recall also that $\gamma_{0}=3 / 10$, as indicated above. Note that condition (35) can be slightly improved by replacing $\gamma_{0}$ by the constant

$$
\gamma_{*} \approx 0.2927
$$

(see $[1,3]$ for more details). The unpleasant side effect is, however, that estimates (36) and (37) under such a condition are established for $m$ sufficiently large only, which puts an obstacle in obtaining efficient solvability conditions in Corollary 8 below. This circumstance 
is not actually of primary importance since the very aim of the interval halving technique discussed here is to weaken assumption (35) by half and, in any case, the difference between the two conditions is quite insignificant because $\gamma_{0}-\gamma_{*} \approx 0.00727$.

Remark 3 It follows from [3, Lemma 3.12] that estimates (36) and (37) can be shown to hold for all $m \geq 0$ if the definition of functions $\bar{\alpha}_{1}$ and $\overline{\bar{\alpha}}_{1}$ is changed slightly (namely, the multiplier 10/9 is added on the right-hand side of (32), (33)).

It should be mentioned that assumption (35), which, by Theorem 2, ensures the applicability of the iteration scheme based on formulae (19), (20), is twice as weak as assumption (11) for the original sequence (4). The same kind of improvement is achieved concerning the condition on the set $D$ where $f$ is Lipschitzian since, for the scheme without interval halving, one would require that

$$
\exists \varrho: \quad \varrho \geq \frac{p}{4} \delta_{\Omega_{\varrho}}(f)
$$

which is twice as strong as (34). In contrast to the related assumptions from [1] and earlier works, condition (34) is easier to verify because in order to do so one has only to find the value $\delta_{\Omega_{\ell}}(f)$, which is computed directly by estimating $f$. In addition, it is possible to estimate this value in certain cases where some further information on the behaviour of $f$ is known.

Comparing Theorem 2 with Theorems 6.1 and 6.3 of [1], where the values in (6.5) and (6.12) are computed over the entire domain where $f$ is Lipschitzian, we see that the values $\delta_{[0, p / 2], \Omega_{\varrho}}(f)$ and $\delta_{[p / 2, p], \Omega_{\varrho}}(f)$ in Theorem 2 are computed over $\Omega_{\varrho}$ only.

The proof of Theorem 2 is carried out by a suitable modification of that of [1, Theorem 6.5] and is based upon the following lemmata.

Lemma 4 ([1, Lemma 7.1]) Let $x:[0, p / 2] \rightarrow \mathbb{R}^{n}$ and $y:[p / 2, p] \rightarrow \mathbb{R}^{n}$ be arbitrary functions such that $\{x(t): t \in[0, p / 2]\} \subset \Omega$ and $\{y(t): t \in[p / 2, p]\} \subset \Omega$. Then

$$
\begin{aligned}
\left|P_{0} f(\cdot, x(\cdot))\right|(t) & \leq \frac{1}{2} \bar{\alpha}_{1}(t) \delta_{[0, p / 2], \Omega}(f) \\
& \leq \frac{p}{8} \delta_{[0, p / 2], \Omega}(f)
\end{aligned}
$$

for $t \in[0, p / 2]$ and

$$
\begin{aligned}
\left|P_{1} f(\cdot, y(\cdot))\right|(t) & \leq \frac{1}{2} \overline{\bar{\alpha}}_{1}(t) \delta_{[p / 2, p], \Omega}(f) \\
& \leq \frac{p}{8} \delta_{[p / 2, p], \Omega}(f)
\end{aligned}
$$

for $t \in[p / 2, p]$.

In (40), (41), the mappings $P_{0}$ and $P_{1}$ are defined by the equality

$$
\left(P_{i} v\right)(t):=\int_{\frac{i}{2} p}^{t} v(s) d s-\left(\frac{2 t}{p}-i\right) \int_{\frac{i}{2} p}^{\frac{i+1}{2} p} v(s) d s
$$

for all $t \in\left[\frac{i}{2} p, \frac{1}{2}(i+1) p\right], i \in\{0,1\}$, and $v \in C\left(\left[\frac{i}{2} p, \frac{1}{2}(i+1) p\right], \mathbb{R}^{n}\right)$. 
Lemma 5 Let $\varrho$ be a vector satisfying relation (34). Then, for arbitrary $m \geq 0$ and $(\xi, \eta) \in$ $\Omega^{2}$, the inclusions

$$
\left\{x_{m}(t, \xi, \eta): t \in[0, p / 2]\right\} \subset \Omega_{\varrho}
$$

and

$$
\left\{y_{m}(t, \xi, \eta): t \in[p / 2, p]\right\} \subset \Omega_{\varrho}
$$

hold.

Proof Let $\xi$ and $\eta$ be arbitrary vectors from $\Omega$. It is natural to argue by induction. Since $\Omega$ is assumed to be convex, it follows from (17) that $x_{0}(t, \xi, \eta) \in \Omega$ for any $t \in[0, p / 2]$ and $y_{0}(t, \xi, \eta) \in \Omega$ for any $t \in[p / 2, p]$, i.e., (43) and (44) are true for $m=0$. Let us assume that (43) and (44) hold for a certain $m=m_{0}$.

Considering relations (17), (19), (8), (34) and (42) and using Lemma 4, we obtain

$$
\begin{aligned}
\left|x_{m_{0}+1}(t, \xi, \eta)-x_{0}(t, \xi, \eta)\right| & =\left|P_{0} f\left(\cdot, x_{m_{0}}(\cdot, \xi, \eta)\right)\right|(t) \\
& \leq \frac{p}{8} \delta_{[0, p / 2], \Omega}(f) \\
& \leq \varrho
\end{aligned}
$$

for $t \in[0, p / 2]$ and

$$
\begin{aligned}
\left|y_{m_{0}+1}(t, \xi, \eta)-y_{0}(t, \xi, \eta)\right| & =\left|P_{1} f\left(\cdot, y_{m_{0}}(\cdot, \xi, \eta)\right)\right|(t) \\
& \leq \frac{p}{8} \delta_{[p / 2, p], \Omega}(f) \\
& \leq \varrho
\end{aligned}
$$

for $t \in[p / 2, p]$. Since (43) and (44) are satisfied for $m=0$, we see from (45), (46) that all the values of $x_{m_{0}+1}(\cdot, \xi, \eta)$ and $y_{m_{0}+1}(\cdot, \xi, \eta)$ are contained in a $\varrho$-neighbourhood of a point from $\Omega$, which means that (43) and (44) hold for $m=m_{0}+1$. It now remains to use the arbitrariness of $m_{0}$.

The assertion of Theorem 2 is now obtained by replacing [1, Lemma 7.2] by Lemma 5 and arguing by analogy to the proof of Theorems 6.1 and 6.3 from [1]. Furthermore, similarly to [1], using Proposition 1 and Theorem 2, one arrives at the following.

Theorem 6 Assume that $f(t, \cdot) \in \operatorname{Lip}_{K}\left(\Omega_{\varrho}\right)$ for a.e. $t \in[0, p]$, where $\varrho$ is a vector with property (34) and K satisfies condition (35). Then, for every solution $u(\cdot)$ of problem (1), (2) with the property

$$
\{u(t) \mid t \in[0, p]\} \subset \Omega_{\varrho} \text { and }\left\{u(0), u\left(\frac{p}{2}\right)\right\} \subset \Omega,
$$

there exists a pair $\left(\xi_{0}, \eta_{0}\right)$ in $\Omega^{2}$ such that $u(\cdot)=u_{\infty}\left(\cdot, \xi_{0}, \eta_{0}\right)$. On the other hand, the function $u_{\infty}(\cdot, \xi, \eta)$ is a solution of the periodic boundary value problem (1), (2) if and only if the pair 


$$
\begin{gathered}
\Xi(\xi, \eta)=0, \\
\mathrm{H}(\xi, \eta)=0 .
\end{gathered}
$$

Recall that the functions $\Xi: \Omega^{2} \rightarrow \mathbb{R}^{n}$ and $\mathrm{H}: \Omega^{2} \rightarrow \mathbb{R}^{n}$ are defined according to equalities (26) and (30), and the latter equalities make sense in view of Theorem 2.

\section{Constructive solvability analysis}

Theorem 6 provides one a formal reduction of the periodic problem (1), (2) to the system of $2 n$ numerical equations (48) in the sense that the initial data $(u(0), u(p / 2))$ of any solution of (1), (2) with properties (47) can be found from (48). Thus, under the conditions assumed, the question on solutions of the periodic boundary value problem (1), (2) can be replaced that of the system of numerical equations (48). A combination of Proposition 11 and Theorems 2, 6 then suggests one a scheme of investigation of the periodic boundary value problem (1), (2). The practical realisation of the scheme is based upon the so-called approximate determining functions

$$
\begin{aligned}
& \Xi_{m}(\xi, \eta):=\eta-\xi-\int_{0}^{\frac{p}{2}} f\left(\tau, x_{m}(\tau, \xi, \eta)\right) d \tau, \\
& \mathrm{H}_{m}(\xi, \eta):=\xi-\eta-\int_{\frac{p}{2}}^{p} f\left(\tau, y_{m}(\tau, \xi, \eta)\right) d \tau,
\end{aligned}
$$

considered for a fixed value of $m$ and, thus, computable explicitly. Then, as in [1], the function

$$
u_{m}(t, \xi, \eta):= \begin{cases}x_{m}(t, \xi, \eta) & \text { if } t \in[0, p / 2] \\ y_{m}(t, \xi, \eta) & \text { if } t \in(p / 2, p]\end{cases}
$$

can be used to obtain the $m$ th approximation to a solution of problem (1), (2) provided that we are able to find certain $\xi$ and $\eta$ satisfying the $m$ th approximate determining equations

$$
\begin{aligned}
& \Xi_{m}(\xi, \eta)=0, \\
& \mathrm{H}_{m}(\xi, \eta)=0 .
\end{aligned}
$$

Furthermore, it turns out that, under natural conditions, the solvability of the periodic problem (1), (2) can be derived from that of system (52). More precisely, putting

$$
\Phi_{m}(\xi, \eta):=\left(\begin{array}{l}
\eta-\xi-\frac{1}{2} \int_{0}^{p} f\left(\frac{p-\tau}{2}, x_{m}\left(\frac{p-\tau}{2}, \xi, \eta\right)\right) d \tau \\
\xi-\eta-\frac{1}{2} \int_{0}^{p} f\left(\frac{p+\tau}{2}, y_{m}\left(\frac{p+\tau}{2}, \xi, \eta\right)\right) d \tau
\end{array}\right)
$$

and

$$
\Phi_{\infty}(\xi, \eta):=\left(\begin{array}{l}
\eta-\xi-\frac{1}{2} \int_{0}^{p} f\left(\frac{p-\tau}{2}, x_{\infty}\left(\frac{p-\tau}{2}, \xi, \eta\right)\right) d \tau \\
\xi-\eta-\frac{1}{2} \int_{0}^{p} f\left(\frac{p+\tau}{2}, y_{\infty}\left(\frac{p+\tau}{2}, \xi, \eta\right)\right) d \tau
\end{array}\right)
$$

for any $(\xi, \eta) \in \Omega^{2}$, we can state the following. 
Theorem 7 Let $f(t, \cdot) \in \operatorname{Lip}_{K}\left(\Omega_{\varrho}\right)$ for a.e. $t \in[0, p]$, where $\varrho$ is a vector with property (34) and $K$ satisfies condition (35). Moreover, assume that $\Phi_{m}$ satisfies the condition

$$
\operatorname{deg}\left(\Phi_{m}, \Omega\right) \neq 0
$$

for a certain fixed $m \geq 0$ and there exists a continuous mapping $Q:[0,1] \times \Omega^{2}$ which does not vanish on $(0,1) \times \partial \Omega^{2}$ and is such that $Q(0, \cdot)=\Phi_{m}, Q(1, \cdot)=\Phi_{\infty}$. Then there exists a pair $\left(\xi^{*}, \eta^{*}\right) \in \Omega^{2}$ such that the function $u:=u_{\infty}\left(\cdot, \xi^{*}, \eta^{*}\right)$ is a solution of the periodic boundary value problem (1), (2) possessing properties (47).

It should be noted that the vector field $\Phi_{m}$ is finite-dimensional and, thus, the degree involved in (55) is the Brower degree.

Proof We can rely on the argument from the proof of [1, Theorem 6.5]. Indeed, a certain computation based on (53) shows that

$$
\Phi_{m}(\xi, \eta)=\left(\begin{array}{c}
\Xi_{m}(\xi, \eta) \\
\mathrm{H}_{m}(\xi, \eta)
\end{array}\right)
$$

for all $(\xi, \eta) \in \Omega^{2}$ and, thus, (52) is necessary and sufficient for $(\xi, \eta)$ to be a singular point of $\Phi_{m}$. Similarly to [1], the assumptions of the theorem then allow one to construct a nondegenerate deformation of $\Phi_{m}$ into the vector field

$$
(\xi, \eta) \ni \Omega^{2} \mapsto\left(\begin{array}{c}
\Xi(\xi, \eta) \\
\mathrm{H}(\xi, \eta)
\end{array}\right)
$$

the singular points of which determine solutions of problem (1), (2) satisfying condition (47), and use the homotopy invariance of the degree. The remaining property in (47) is a consequence of Lemma 5.

Let the binary relation $\triangleright_{S}$ be defined [3] for any $S \subset \mathbb{R}^{2 n}$ as follows: functions $g=\left(g_{i}\right)_{i=1}^{2 n}$ : $\mathbb{R}^{2 n} \rightarrow \mathbb{R}^{2 n}$ and $h=\left(h_{i}\right)_{i=1}^{2 n}: \mathbb{R}^{2 n} \rightarrow \mathbb{R}^{2 n}$ are said to satisfy the relation $g \triangleright_{S} h$ if and only if there exists a function $v: S \rightarrow\{1,2, \ldots, 2 n\}$ such that $g_{v(z)}(z)>h_{v(z)}(z)$ at every point $z \in S$. Using this relation, one can formulate an efficient condition sufficient for the solvability of problem (1), (2).

Corollary 8 Let $f(t, \cdot) \in \operatorname{Lip}_{K}\left(\Omega_{\varrho}\right)$ for a.e. $t \in[0, p]$, where $\varrho$ satisfies inequality (34) and $K$ has property (35). Let, moreover,

$$
\left|\Phi_{m}\right| \triangleright_{\partial \Omega} \frac{5 p}{18}\left(\begin{array}{l}
M_{m} \delta_{[0, p / 2], \Omega}(f) \\
M_{m} \delta_{[p / 2, p], \Omega}(f)
\end{array}\right)
$$

for a certain fixed $m \geq 2$, where

$$
M_{m}:=\left(\frac{\gamma_{0} p}{2}\right)^{m+1} K^{m+1}\left(\mathbf{1}_{n}-\frac{\gamma_{0} p}{2} K\right)^{-1} .
$$

Then there exists a pair $\left(\xi^{*}, \eta^{*}\right) \in \Omega^{2}$ such that $u:=u_{\infty}\left(\cdot, \xi^{*}, \eta^{*}\right)$ is a solution of problem (1), (2) possessing properties (47). 
Proof It is sufficient to apply Theorem 7 with the linear homotopy

$$
Q(\theta, \xi, \eta):=(1-\theta) \Phi_{m}(\xi, \eta)+\theta \Phi_{\infty}(\xi, \eta)
$$

for $(\xi, \eta) \in \Omega^{2}, \theta \in[0,1]$, and use estimate (10.11) from [1].

Recall that $\gamma_{0}$ in (58) is given by (12). It is important to emphasise that conditions of Corollary 8 are assumed for a fixed $m$, and all the values depending on it are evaluated in finitely many steps.

The next assertion is interesting especially because it is, in fact, based upon properties of the starting approximation and, thus, shows how a useful information can be obtained when no iterations have been carried out at all. Note that the zeroth approximation is very rough indeed in any case: the periodic solution is approximated by a piecewise linear function (see Figure 6).

With the given function $f$ involved in (1), we associate the function $f^{\#}: \Omega^{2} \rightarrow \mathbb{R}^{2 n}$ by putting

$$
f^{\#}(\xi, \eta):=\left(\begin{array}{l}
\eta-\xi-\frac{1}{2} \int_{0}^{p} f\left(\frac{p-\tau}{2}, \frac{\tau}{p} \xi+\left(1-\frac{\tau}{p}\right) \eta\right) d \tau \\
\xi-\eta-\frac{1}{2} \int_{0}^{p} f\left(\frac{p+\tau}{2}, \frac{\tau}{p} \xi+\left(1-\frac{\tau}{p}\right) \eta\right) d \tau
\end{array}\right)
$$

for any $(\xi, \eta) \in \Omega^{2}$. Note that, unlike $f$, the function $f^{\#}$ depends on the phase variables only.

Corollary 9 Assume that there is a $\varrho$ with property (47) and $f(t, \cdot) \in \operatorname{Lip}_{K}\left(\Omega_{\varrho}\right), t \in[0, p]$, with $K$ satisfying inequality (35). Let, furthermore,

$$
\operatorname{deg}\left(f^{\#}, \Omega\right) \neq 0
$$

and

$$
\left|f^{\#}\right| \triangleright_{\partial \Omega} \frac{5 p^{2}}{108}\left(\begin{array}{l}
K\left(\mathbf{1}_{n}-\frac{\gamma_{0} p}{2} K\right)^{-1} \delta_{\left[0, \frac{1}{2} p\right], D}(f) \\
K\left(\mathbf{1}_{n}-\frac{\gamma_{0} p}{2} K\right)^{-1} \delta_{\left[\frac{1}{2} p, p\right], D}(f)
\end{array}\right) .
$$

Then the p-periodic problem (1), (2) has at least one solution $u(\cdot)$ which possesses properties (47).

Proof Equalities (17), (18), (53) and (60) imply that $f^{\#}=\Phi_{0}$. It is also easy to verify by computation that condition (62) can be rewritten in the form

$$
\left|\Phi_{0}\right| \triangleright_{\partial \Omega} \frac{5 p}{18}\left(\begin{array}{c}
\tilde{M}_{0} \delta_{[0, p / 2], \Omega}(f) \\
\tilde{M}_{0} \delta_{[p / 2, p], \Omega}(f)
\end{array}\right)
$$

where $\tilde{M}_{0}:=(10 / 9) M_{0}$ and $M_{0}$ is given by (58). Arguing similarly to [1, Lemma 9.2] and Corollary 8 and taking Remark 3 into account, one can show that (63) ensures the nondegeneracy of homotopy (59). The required conclusion then follows from Theorem 7. 


\section{Discussion}

Theorems of the kind specified above allow one to study the periodic problem (1), (2) following the lines of $[1,3]$. This analysis is constructive in the sense that the assumptions can be verified efficiently and the results of computation, regarded at first only as candidates for approximate solutions, simultaneously open a way to prove the solvability in a rigorous manner. As regards the computation of iterations themselves, it is helpful to apply suitable simplified versions of the algorithm, not discussed here, which are better adopted for use with computer algebra systems. The use of polynomial approximations under similar circumstances was considered, in particular, in [7].

It is interesting to note that $f^{\#}$ involved in Corollary 9 can be considered as a 'halved' analogue of the averaged map

$$
\bar{f}(\xi):=\int_{0}^{p} f(s, \xi) d s
$$

for $x \in \Omega$, which arises similarly to $f^{\#}$ in the situation where no interval halving is carried out. In the latter case, one has the following statement, which is a reformulation of $[1$, Corollary 13.2].

Corollary 10 Let there exist some @ with property (34). Let

$$
\operatorname{deg}(\bar{f}, \Omega) \neq 0
$$

and $f(t, \cdot) \in \operatorname{Lip}_{K}\left(\Omega_{\varrho}\right), t \in[0, p]$, with $K$ satisfying inequality (35). If

$$
|\bar{f}| \triangleright_{\partial \Omega} \frac{5 p^{2}}{27} K\left(\mathbf{1}_{n}-\gamma_{0} p K\right)^{-1} \delta_{\Omega_{\varrho}}(f),
$$

then the p-periodic problem (1), (2) has a solution $u(\cdot)$ with properties (47).

Assumption (65) with $\bar{f}$ given by (64) arises frequently in topological continuation theorems where the homotopy to the averaged equation is considered (see, e.g., $[8,9]$ ).

It should also be noted that, as a natural extension of the above said, one can consider a scheme with multiple interval divisions. Although the addition of intermediate nodes increases the number of equations to be solved numerically (at $k_{0}$ interval halvings, one ultimately arrives a system of $2^{k_{0}}$ determining equation with respect to $2^{k_{0}}$ variables), the important gain is the ability to apply the method regardless of the value of the Lipschitz constant.

The construction of such a scheme is based on the appropriate modification of the initial approximation, which will then depend on more parameters. Consider, e.g., the transition from $k_{0}=1$ to $k_{0}=2$. Renaming the variables as $\xi=\left(\xi_{-1}, \xi_{1}\right)$ in the former case for more convenience and denoting the initial approximation by $u_{0}(\cdot, \xi)$, we rewrite (21), (22) in the form

$$
u_{0}(0, \xi)=\xi_{-1}, \quad u_{0}\left(\frac{p}{2}, \xi\right)=\xi_{1}, \quad u_{0}(p, \xi)=\xi_{-1} .
$$

Thus, the initial approximation $u_{0}(\cdot, \xi)$ in the corresponding iteration scheme with one division is the linear function joining the points $\left(0, \xi_{-1}\right),\left(\frac{p}{2}, \xi_{1}\right)$ and $\left(p, \xi_{-1}\right)$. Extending this 
tree graph like notation to the case of two interval halvings $\left(k_{0}=2\right)$ and arguing similarly, we arrive at the following equalities determining $u_{0}(\cdot, \xi)$ :

$$
\begin{array}{ll}
u_{0}(0, \xi)=\xi_{-1,-1}, & u_{0}\left(\frac{p}{4}, \xi\right)=\xi_{-1,1}, \quad u_{0}\left(\frac{p}{2}, \xi\right)=\xi_{1,-1}, \\
u_{0}\left(\frac{3 p}{4}, \xi\right)=\xi_{1,1}, \quad u_{0}(p, \xi)=\xi_{-1,-1} .
\end{array}
$$

In other words, relations $(68)$ mean that the function $u_{0}(\cdot, \xi)$ for $k_{0}=2$ depends on the array of parameters $\xi=\left(\xi_{-1,-1}, \xi_{-1,1}, \xi_{1,-1}, \xi_{1,1}\right)$ and is obtained by the linear interpolation of the points $\left(0, \xi_{-1,-1}\right),\left(\frac{p}{4}, \xi_{-1,1}\right),\left(\frac{p}{2}, \xi_{1,-1}\right),\left(\frac{p}{2}, \xi_{1,-1}\right),\left(\frac{3 p}{4}, \xi_{1,1}\right)$ and $\left(p, \xi_{-1,-1}\right)$. For $k_{0} \geq 3$, the structure of $u_{0}(\cdot, \xi)$ is completely analogous, the idea is clear from Table 1 and Figure 6: one simply draws a broken line joining the corresponding nodes. Once $u_{0}(\cdot, \xi)$ is constructed, the formulae for the subsequent approximations are derived automatically by rescaling the projection map to the corresponding subintervals (we do not need the corresponding explicit formulae here and, therefore, omit the details).

This observation leads one to the following algorithm of investigation of the periodic problem (1), (2):

Table 1 Variables involved in the determining equations for the respective number of interval halvings

\begin{tabular}{ll}
\hline $\boldsymbol{k}_{\mathbf{0}}$ & Variables in the determining equations \\
\hline 0 & $\xi$ \\
1 & $\xi_{-1}, \xi_{1}$ \\
2 & $\xi_{-1,-1}, \xi_{-1,1}, \xi_{1,-1}, \xi_{1,1}$ \\
3 & $\xi_{-1,-1,-1}, \xi_{-1,-1,1}, \xi_{-1,1,-1}, \xi_{-1,1,1}, \xi_{1,-1,-1}, \xi_{1,-1,1}, \xi_{1,1,-1}, \xi_{1,1,1}$ \\
$\ldots$ & $\ldots$ \\
\hline
\end{tabular}

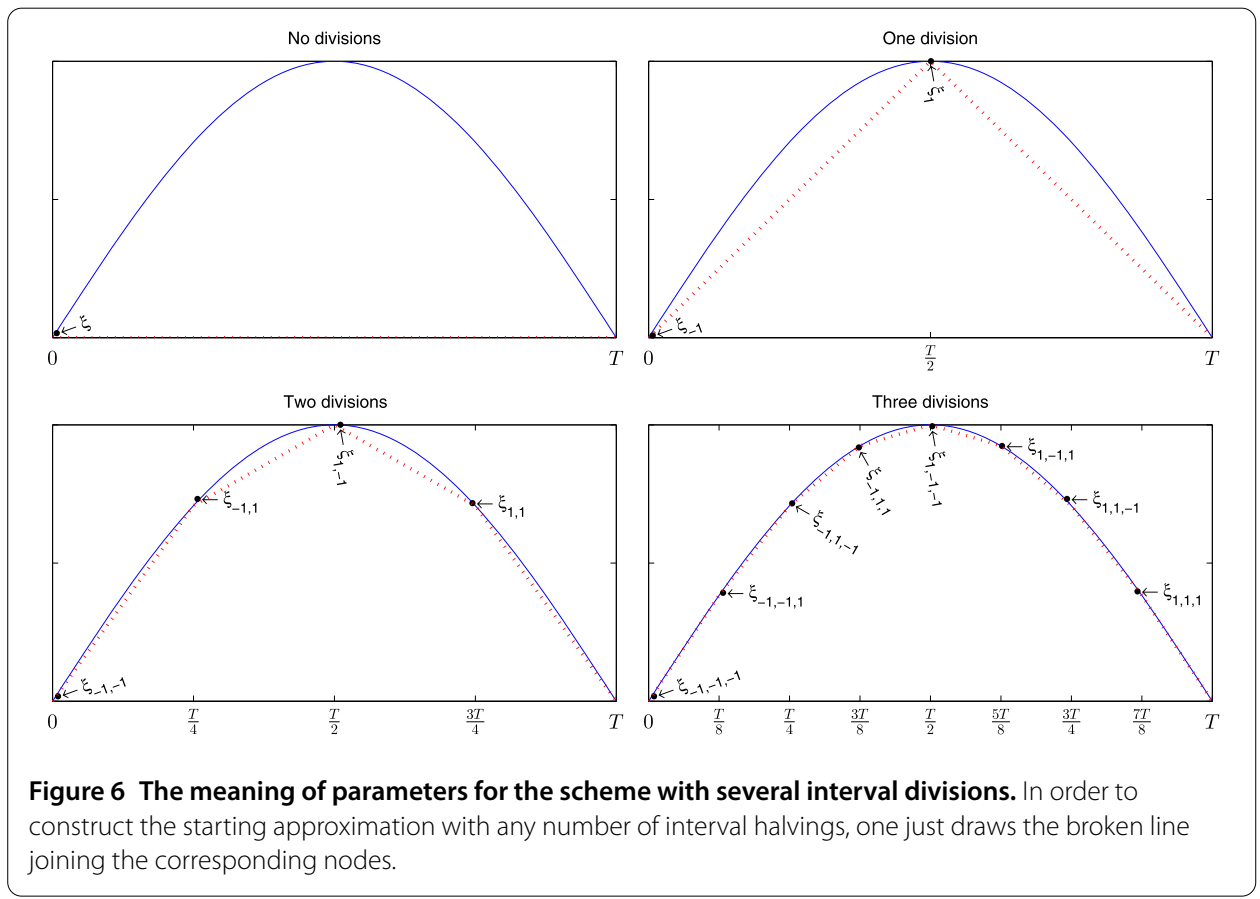


1. Fix a certain $k_{0}$ and consider the scheme with $k_{0}$ interval divisions. Fix an $m_{0}$ and construct $u_{m}(\cdot, \xi)$ for $m=0,1, \ldots, m_{0}$.

2. Solve the $m$ th approximate determining equations for $\xi$, find a root $\xi^{[m]}$, and put

$$
U_{m}(t):=u_{m}\left(t, \xi^{[m]}\right), \quad t \in[0, p], m=0,1, \ldots, m_{0} .
$$

In the case the equation has multiple roots, (69) and the related analysis are repeated for each of them (one can study multiple solutions of the original problem in this way).

3. 'Check' the behaviour of the functions $U_{0}, U_{1}, \ldots, U_{m_{0}}$ (the heuristic step). If promising (i.e., there are some signs of convergence), choose a suitable $\Omega$ containing the graph of $U_{m_{0}}$, find a $\varrho$ from the condition

$$
\varrho \geq \frac{p}{2^{k_{0}+2}} \delta_{\Omega_{\varrho}}(f)
$$

compute the Lipschitz matrix $K$ for $f$ in $\Omega_{\varrho}$, and verify the convergence condition

$$
r(K)<\frac{2^{k_{0}}}{\gamma_{0} p} .
$$

If not successful with either (70) or (71), increase $k_{0}$ appropriately and try again.

4. Verify conditions of the existence theorem for $\Omega$ and $m_{0}$. If not satisfied, or if the precision of $U_{m_{0}}$ is insufficient, pass to $m=m_{0}+1$ and study $U_{m_{0}+1}$. Otherwise the algorithm stops, and the outcome is:

(a) there is a solution $u$ of (1), (2) and $u \approx U_{m_{0}}$;

(b) $\exists\left(\xi_{*}, \eta_{*}\right) \in \Omega^{2}: u(\cdot)=u_{\infty}\left(\cdot, \xi_{*}, \eta_{*}\right)$;

(c) the space localisation of the graph of $u$ is described by properties (47).

Note the role of interval divisions in the algorithm: for $K$ not satisfying the smallness condition (11) and $k_{0}=0$ (i.e., when $u_{m}$ is constructed according to (4) without any interval divisions), the algorithm would stop at step 3 without any result. However, it is obvious that (70) and (71) are both satisfied if $k_{0}$ is chosen to be large enough.

In relation to the last remark, it is interesting to compare the approach discussed here with the Cesari method [10], which likewise provides one a way to reduce the periodic problem (1), (2) to a system of finitely many numerical equations. The idea of construction of the iterations there is based, in the notation of [11], on the use of the operator

$$
H_{m} u:=L-P_{m} L
$$

in a suitable space of $p$-periodic functions, where

$$
(L y)(t):=\int_{0}^{t} y(s) d s-\frac{t}{p} \int_{0}^{p} y(s) d s, \quad t \in[0, p],
$$

$m$ is fixed, and $P_{m}$ stands for the $m$ th partial sum of the Fourier series of the corresponding function. There are visible similarities between the two approaches and, most importantly, the scheme of Cesari is also proved to be applicable regardless on the smallness of the Lipschitz constant (see [11]). The number of resulting determining equations therewith 
depends on the Lipschitz constant of $f$ as well (in fact, it grows with $m$, the convergence being guaranteed by suitable properties of $H_{m}$ for $m$ large enough), which reminds us of Table 1 in our case. The approach presented in this note, in our opinion, has the advantage that, firstly, the computation of iterations is significantly simpler (apart of the integral mean, one does not need to compute any higher order terms in the Fourier expansion) and, secondly, it can be used for other problems as well, whereas, due to the nature of formula (72), the use of Cesari's scheme is limited to periodic functions.

In particular, the method described above is rather easy to adopt for application to twopoint boundary value problems different from the periodic ones. Indeed, consider the problem with linear two-point conditions where one of the coefficient matrices is nonsingular. Without loss of generality, we can assume that the problem has the form

$$
\begin{aligned}
& u^{\prime}(t)=f(t, u(t)), \quad t \in[0, p], \\
& u(p)-A u(0)=c,
\end{aligned}
$$

where $A$ is a square matrix of dimension $n$ (possibly, singular), $c \in \mathbb{R}^{n}, f:[0, p] \times \mathbb{R}^{n} \rightarrow \mathbb{R}^{n}$, and $p \in(0, \infty)$.

The transition from the periodic problem (1), (2) to problem (73), (74) is then surprisingly simple: one does not need but to adjust the functions $x_{0}(\cdot, \xi, \eta)$ and $y_{0}(\cdot, \xi, \eta)$ so that they satisfy the boundary condition (74). More precisely, let us fix a suitable $\Omega$ and take arbitrary $\xi$ and $\eta$ in it. Introduce the sequences of functions $x_{m}:[0, p / 2] \times \Omega^{2} \rightarrow \mathbb{R}^{n}$ and $y_{m}:[p / 2, p] \times \Omega^{2} \rightarrow \mathbb{R}^{n}, m=0,1, \ldots$, according to the same recurrence formulae as in (19), (20), where, instead of (17), (18), the functions $x_{0}(\cdot, \xi, \eta)$ and $y_{0}(\cdot, \xi, \eta)$ are given by the equalities

$$
\begin{aligned}
& x_{0}(t, \xi, \eta):=\left(1-\frac{2 t}{p}\right) \xi+\frac{2 t}{p} \eta, \quad t \in[0, p / 2], \\
& y_{0}(t, \xi, \eta):=2\left(1-\frac{t}{p}\right) \eta+\left(\frac{2 t}{p}-1\right)(A \xi+c), \quad t \in[p / 2, p] .
\end{aligned}
$$

Clearly, $x_{0}(\cdot, \xi, \eta)$ and $y_{0}(\cdot, \xi, \eta)$ given by $(75),(76)$ are the linear functions satisfying the equalities

$$
\begin{array}{ll}
x_{0}(0, \xi, \eta)=\xi, & x_{0}\left(\frac{p}{2}, \xi, \eta\right)=\eta, \\
y_{0}\left(\frac{p}{2}, \xi, \eta\right)=\eta, & y_{0}(p, \xi, \eta)=A \xi+c,
\end{array}
$$

which reduce to (17), (18) if $A$ is the unit matrix and $c=0$. Then, similarly to Proposition 1 , it is not difficult to prove the following.

Proposition 11 Let $(\xi, \eta) \in \Omega^{2}$ be fixed. If the limits $x_{\infty}(\cdot, \xi, \eta)$ and $y_{\infty}(t, \xi, \eta)$ of sequences (19) and (20) exist, then:

1. The function $x_{\infty}(\cdot, \xi, \eta)$ has the property

$$
x_{\infty}\left(\frac{p}{2}, \xi, \eta\right)-A x_{\infty}(0, \xi, \eta)=\eta-A \xi
$$

and is the unique solution of the initial value problem (24), (25) with $\Xi$ given by (26). 
2. The function $y_{\infty}(\cdot, \xi, \eta)$ has the property

$$
y_{\infty}(p, \xi, \eta)-A y_{\infty}\left(\frac{p}{2}, \xi, \eta\right)=A(\xi-\eta)+c
$$

and is the unique solution of the initial value problem (28), (29) with $\mathrm{H}$ given by (30).

We see from Proposition 11 that properties of sequences $x_{m}(\cdot, \xi, \eta), y_{m}(\cdot, \xi, \eta), m \geq 0$, constructed for problem (73), (74) are rather similar to those for the periodic problem (1), (2) (in particular, the definition of functions $\Xi$ and $H$ is the same as in Proposition 1). In both cases, the iteration is carried out according to formulae (19), (20), the only difference being in equalities (75), (76) for $x_{0}(\cdot, \xi, \eta)$ and $y_{0}(\cdot, \xi, \eta)$. As a result, the corresponding limit functions satisfy the boundary conditions (79), (80).

Based on Proposition 11, one can develop essentially the same techniques that have been indicated above for the periodic problem (1), (2). The main difference in the proofs is that, in addition to guaranteeing that the appropriate values $\xi$ should belong to $\Omega$, we also have to ensure that $A \xi+c \in \Omega$ as well. The convergence of iterations is then guaranteed for all $\eta$ from the set $\Omega$ and $\xi$ belonging to its subset $S_{A, c}(\Omega)$ defined by the relation

$$
S_{A, c}(\Omega):=\{\xi \in \Omega: A \xi+c \in \Omega\} .
$$

Clearly, $S_{A, c}(\Omega)$ is the union of all the subsets of $\Omega$ invariant with respect to the transformation $x \mapsto A x+c$. For example, if $\Omega$ is a set on the plane $(n=2)$ containing the origin, then $S_{\left(\begin{array}{ll}0 & 1 \\ 1 & 0\end{array}\right), 0}(\Omega)$ is the part of $\Omega$ that is symmetric with respect to the diagonal passing through the first and the third quadrants (see Figure 7).

Theorem 12 Let there exist a non-negative vector $\varrho$ with property (34) such that $f(t, \cdot) \in$ $\operatorname{Lip}_{K}\left(\Omega_{\varrho}\right)$ for a.e. $t \in[0, p]$ with a certain matrix $K$ satisfying inequality (35). Then, for

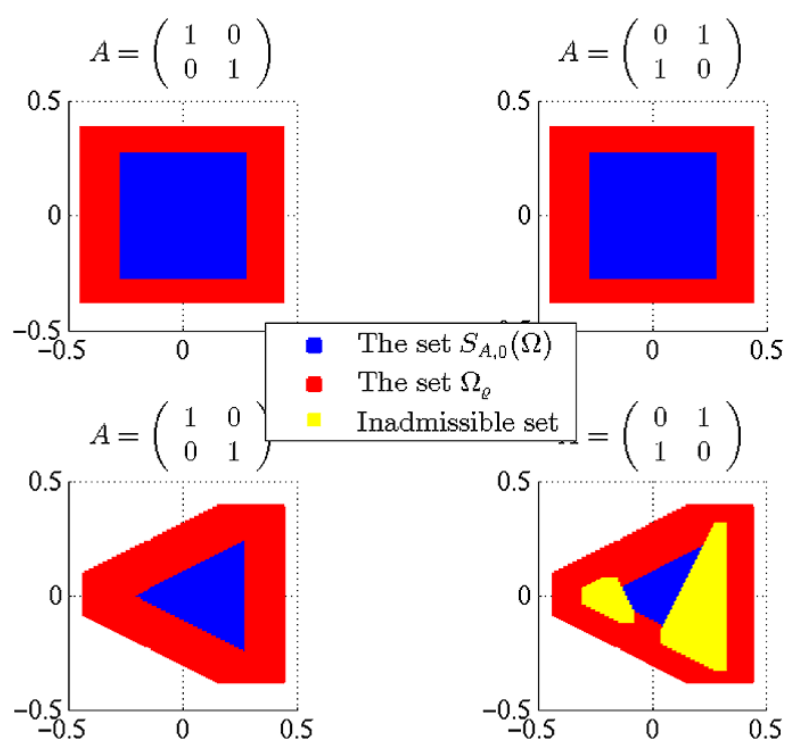

Figure 7 The sets $S_{A, c}(\Omega)$ and $\Omega_{\varrho}$ in examples, $\varrho=\operatorname{col}(1 / 8,1 / 16)$. 
all fixed $(\xi, \eta) \in S_{A, c}(\Omega) \times \Omega$, the sequences $\left\{x_{m}(\cdot, \xi, \eta): m \geq 0\right\}$ and $\left\{y_{m}(\cdot, \xi, \eta): m \geq 0\right\}$ with $x_{0}(\cdot, \xi, \eta), y_{0}(\cdot, \xi, \eta)$ defined by $(75)$ and $(76)$ converge uniformly on the corresponding intervals and, moreover, estimates (36), (37) hold.

The same remark as has been made above concerning Theorem 2 applies to Theorem 12: its assertion remains true if (35) is replaced by the inequality

$$
r(K)<\frac{2}{\gamma_{* p}}
$$

with $\gamma_{*}$ given by (38).

Theorem 12 is easily obtained by analogy to Theorem 2 for the periodic problem. The verification of the conditions of Theorem 12 is also pretty much similar to the latter case. One has to keep in mind that the techniques for the two-point problem (73), (74) are applicable for the values of parameters lying in $S_{A, c}(\Omega) \times \Omega$, and not in the entire $\Omega^{2}$, which is the case in Theorem 2 (unless $A$ is the unit matrix and $c=0$ ). This circumstance has a natural explanation due to (77) and (78), whence one deduces that both $\xi$ and $A \xi+c$ will eventually belong to one and the same set, which fact is then used in Lemma 5 . If, for example, $c$ is equal to zero and

$$
A=\frac{1}{\sqrt{2}}\left(\begin{array}{cc}
1 & -1 \\
1 & 1
\end{array}\right),
$$

then the assertion of Theorem 12 is true only for the part of $\Omega$ that is invariant under the rotation by $45^{\circ}$ counter-clockwise. In this way, e.g., Figure 5 is replaced by Figure 8 once the two-point problem (73), (74) with $A$ given by (82) is considered. Note that all the sets on Figures 5 and 8 contain the origin, and the yellow regions on the latter one indicate the points from $\Omega$ that cannot be regarded as candidates for initial values of the solution in question.

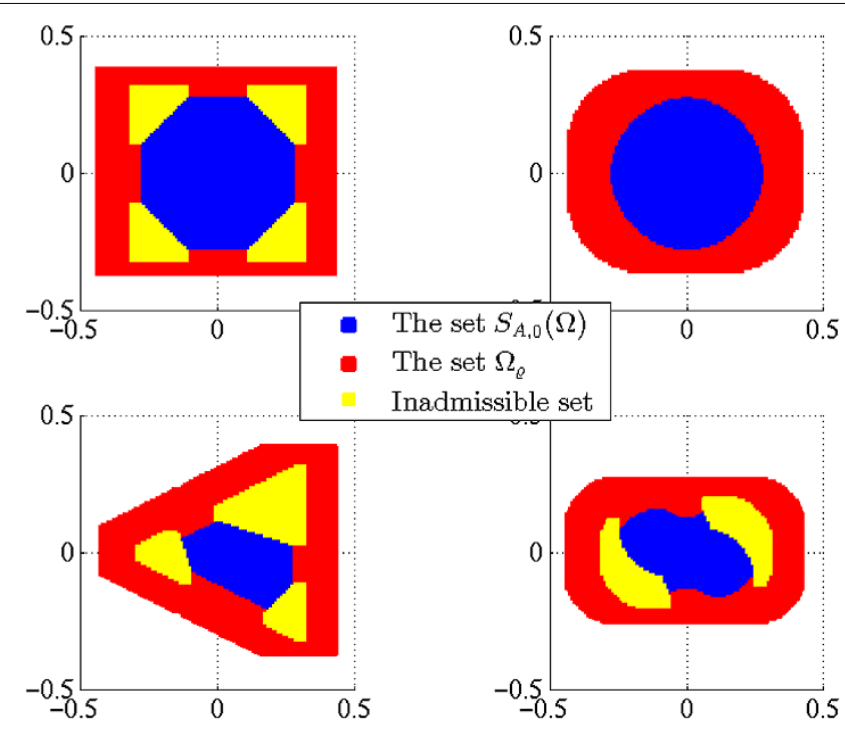

Figure 8 The sets $S_{A, C}(\Omega)$ and $\Omega_{\varrho}$ in examples, $\varrho=\operatorname{col}(1 / 8,1 / 16)$ and $A$ is given by $(82)$. 


\section{Competing interests}

The authors declare that they have no competing interests.

\section{Authors' contributions}

All the authors contributed equally to the final version of this work and approved its present form.

\section{Author details}

${ }^{1}$ Institute of Mathematics, Academy of Sciences of Czech Republic, 22 Žižkova St., Brno, 616 62, Czech Republic.

${ }^{2}$ Department of Analysis, University of Miskolc, Miskolc-Egyetemváros, Miskolc, 3515, Hungary. ${ }^{3}$ Department of Mathematics, Uzhgorod National University, 46 Pidhirna St., Uzhgorod, 88 000, Ukraine. ${ }^{4}$ Mathematical Institute, Slovak Academy of Sciences, 49 Štefánikova St., Bratislava, 814 73, Slovakia.

\section{Acknowledgements}

The work was supported in part by RVO: 67985840 (A Rontó) and the SAIA National Scholarship Programme of the Slovak Republic (N Shchobak).

Received: 18 March 2014 Accepted: 20 June 2014 Published online: 26 September 2014

\section{References}

1. Rontó, A, Rontó, M, Shchobak, N: Constructive analysis of periodic solutions with interval halving. Bound. Value Probl. 2013, 57 (2013). doi:10.1186/1687-2770-2013-57

2. Samoilenko, AM, Rontó, NI: Numerical-Analytic Methods in the Theory of Boundary-Value Problems for Ordinary Differential Equations. Naukova Dumka, Kiev (1992) (in Russian). Edited and with a preface by YA Mitropolskii

3. Rontó, A, Rontó, M: Successive approximation techniques in non-linear boundary value problems for ordinary differential equations. In: Handbook of Differential Equations: Ordinary Differential Equations, vol. IV, pp. 441-592. Elsevier, Amsterdam (2008)

4. Nirenberg, L: Topics in Nonlinear Functional Analysis. Courant Lecture Notes Series. Am. Math. Soc., New York (1974). With a chapter by E Zehnder, notes by RA Artino, lecture notes, 1973-1974

5. Gaines, RE, Mawhin, JL: Coincidence Degree and Nonlinear Differential Equations. Lecture Notes in Mathematics, vol. 568. Springer, Berlin (1977)

6. Rontó, M, Samoilenko, AM: Numerical-Analytic Methods in the Theory of Boundary-Value Problems. World Scientific, River Edge (2000). doi:10.1142/9789812813602. With a preface by YA Mitropolsky and an appendix by the authors and SI Trofimchuk

7. Rontó, A, Rontó, M, Holubová, G, Nečesal, P: Numerical-analytic technique for investigation of solutions of some nonlinear equations with Dirichlet conditions. Bound. Value Probl. 2011, 58 (2011). doi:10.1186/1687-2770-2011-58

8. Mawhin, J: Topological Degree Methods in Nonlinear Boundary Value Problems. CBMS Regional Conference Series in Mathematics, vol. 40. Am. Math. Soc., Providence (1979). Expository lectures from the CBMS Regional Conference held at Harvey Mudd College, Claremont, Calif., June 9-15, 1977

9. Capietto, A, Mawhin, J, Zanolin, F: A continuation approach to superlinear periodic boundary value problems. J. Differ. Equ. 88(2), 347-395 (1990). doi:10.1016/0022-0396(90)90102-U

10. Cesari, L: Asymptotic Behavior and Stability Problems in Ordinary Differential Equations, 3rd edn. Ergebnisse der Mathematik und Ihrer Grenzgebiete, vol. 16. Springer, New York (1971)

11. Knobloch, H-W: Remarks on a paper of $L$. Cesari on functional analysis and nonlinear differential equations. Mich. Math. J. 10,417-430 (1963)

doi:10.1186/s13661-014-0164-9

Cite this article as: Rontó et al.: Notes on interval halving procedure for periodic and two-point problems. Boundary Value Problems 2014 2014:164.

\section{Submit your manuscript to a SpringerOpen ${ }^{\circ}$ journal and benefit from:}

$\rightarrow$ Convenient online submission

Rigorous peer review

- Immediate publication on acceptance

- Open access: articles freely available online

- High visibility within the field

- Retaining the copyright to your article 